MATHEMATICS OF COMPUTATION

Volume 67, Number 224, October 1998, Pages 1423-1450

S 0025-5718(98)00983-1

\title{
BOUNDS FOR EIGENVALUES AND CONDITION NUMBERS IN THE $p$-VERSION OF THE FINITE ELEMENT METHOD
}

\author{
NING HU, XIAN-ZHONG GUO, AND I. NORMAN KATZ
}

\begin{abstract}
In this paper, we present a theory for bounding the minimum eigenvalues, maximum eigenvalues, and condition numbers of stiffness matrices arising from the $p$-version of finite element analysis. Bounds are derived for the eigenvalues and the condition numbers, which are valid for stiffness matrices based on a set of general basis functions that can be used in the $p$-version. For a set of hierarchical basis functions satisfying the usual local support condition that has been popularly used in the $p$-version, explicit bounds are derived for the minimum eigenvalues, maximum eigenvalues, and condition numbers of stiffness matrices. We prove that the condition numbers of the stiffness matrices grow like $p^{4(d-1)}$, where $d$ is the number of dimensions. Our results disprove a conjecture of Olsen and Douglas in which the authors assert that "regardless of the choice of basis, the condition numbers grow like $p^{4 d}$ or faster". Numerical results are also presented which verify that our theoretical bounds are correct.
\end{abstract}

\section{INTRODUCTION}

In the last fifteen years or so, one of the major advances in the area of finite element analysis has been the development of the $p$-version and the $h-p$ version of the finite element methods. The classical finite element method, also called $h$-version, achieves the accuracy of its finite element solution by decreasing the mesh size $h$ while the degree of the polynomial basis functions is fixed [15]. In the $p$-version, the accuracy of the finite element solution is achieved by increasing the degree $p$ of the polynomial basis functions while the mesh is fixed [1],[2],[20]. The $h-p$ version is the combination of both the $h$-version and the $p$-version [3], [5]. Theoretical analysis and computational practice of the $p$-version of the finite element method have been carried out during the last decade. Currently, the theoretical development and computational practice of the $h-p$ version of the finite element method are active research areas.

By comparing the two methods, namely the $p$-version and the $h$-version, one may notice that for many theoretical results developed in the $h$-version there are corresponding theoretical results in the $p$-version. One example is the convergence of the $h$-version and the $p$-version. Even though there are many such correspondences, there are some results that were developed in the $h$-version, but have not yet

Received by the editor July 15, 1996 and, in revised form, April 1, 1997.

1991 Mathematics Subject Classification. Primary 65N30; Secondary 65N22, 65F33.

Key words and phrases. Eigenvalues; condition number; $p$-version of the finite element method.

This research was supported by Air Force Office of Scientific Research under grant number AFOSR 92-J-0043, and by the National Science Foundation under grant number DMS-9626202. Some of the results presented here are part of the doctoral dissertation of the first author.

(C)1998 American Mathematical Society 
been developed in the $p$-version. One of such missing correspondence is a generic bound for the minimum eigenvalue, maximum eigenvalue, and condition number of a general stiffness matrix arising from the $p$-version of finite element analysis. More specifically, it is well known that in the $h$-version under uniform or quasi-uniform mesh the condition numbers are bounded by $O\left(h^{-2}\right)$ [15]. This result is very useful in many theoretical analyses that relate to the $h$-version. For example, in many convergence analyses of multigrid methods, this result is one of the key elements in deriving convergence factors [9],[10],[11]. Also, the condition number is critical to the convergence of the conjugate gradient algorithms [14].

In the $p$-version, however, to our knowledge there is no general theory to characterize the minimum eigenvalue, maximum eigenvalue, and condition number of a stiffness matrix based on a set of general polynomial basis functions. It is obvious that there are many different types of basis functions that can be used in the $p$ version, such as basis functions based on the Legendre polynomials, basis functions based on Chebyshev polynomials, etc. Therefore, we believe that a general theory concerning the eigenvalues and condition numbers in the $p$-version is not only necessary but also very useful.

In this paper, we present a theory for bounding the eigenvalues and condition number of a general stiffness matrix arising from the $p$-version of finite element analysis. Bounds are derived for the eigenvalues and condition number which are valid for a stiffness matrix based on a set of general basis functions that can be used in the $p$-version. After developing such a general theory, we turn our attention to a special class of basis functions: a set of hierarchical basis functions satisfying the usual local support condition that has been popularly used in the $p$-version [21]. Explicit bounds are derived for the minimum eigenvalues, maximum eigenvalues, and condition numbers of a stiffness matrix based on these basis functions. We prove that the condition numbers of the stiffness matrices grow like $p^{4(d-1)}$, where $d$ is the number of dimensions. Our results disprove a conjecture of Olsen and Douglas [22] in which the authors assert that "regardless of the choice of basis, the condition numbers grow like $p^{4 d}$ or faster".

Maitre and Pourquier [16],[17] have obtained results for condition numbers for the hierarchical basis functions mentioned above. Our results are similar to those in $[16],[17]$, but different in a substantial way. The results in $[16],[17]$ apply only to the internal basis functions (so-called "bubble" modes); ours apply to all basis functions and are therefore applicable to practical finite element analysis, which includes all modes. Also the results in [16] and [17] are for the derivative matrix and mass matrix separately, whereas ours are also for the sum of the derivative matrix and mass matrix.

The paper is organized as follows. Section 2 contains definitions and some preliminary results. Bounds for the minimum eigenvalues, maximum eigenvalues, and condition numbers are derived in Section 3. In Section 4, for a set of hierarchical basis functions, explicit bounds are derived for the eigenvalues and condition numbers. Numerical results are also given in this section.

\section{Preliminary}

Let $\Omega \subset R^{2}$ be a bounded domain with piecewise smooth boundaries $\partial \Omega$. For any integer $k \geq 0$, let $H^{k}(\Omega)$ (or $H_{0}^{k}(\Omega)$ ) be the standard Sobolev space. 
Consider a second order elliptic partial differential equation

$$
\left\{\begin{array}{l}
-\nabla(a \nabla u)+b u=g \text { in } \Omega \\
u=0 \quad \text { on } \Gamma^{D} \\
\frac{\partial u}{\partial n}=h \quad \text { on } \Gamma^{N},
\end{array}\right.
$$

where $\Gamma^{D} \cup \Gamma^{N}=\partial \Omega, a \in C^{1}(\bar{\Omega}), b \in C(\Omega), a(x) \geq \alpha>0, b(x) \geq 0$ and $g \in L^{2}(\Omega)$.

Define

$$
H(\Omega)=\left\{u \mid u \in H^{1}(\Omega), u=0 \text { on } \Gamma^{D}\right\}
$$

and

$$
\begin{gathered}
a(u, v)=\int_{\Omega}(a \nabla u \cdot \nabla v+b u v) d x, \\
f(v)=\int_{\Omega} g v d x+\int_{\Gamma^{N}} h v d s .
\end{gathered}
$$

We assume that the bilinear form $a(u, v)$ satisfies the $V$-ellipticity assumption

$$
a(u, u) \geq C\|u\|_{H^{1}(\Omega)}^{2},
$$

where $C>0$ is a constant.

The exact solution. Find $u \in H(\Omega)$ such that

$$
a(u, v)=f(v)
$$

for all $v \in H(\Omega)$, where $u$ is called the exact solution. The exact solution exists and is unique under common assumptions for $g, h$ and $\partial \Omega$.

Let $K=[-1,1] \times[-1,1]$ be the standard element and let a set of standard polynomial basis functions with degree $\leq p$ be denoted by $\phi$ :

$$
\phi=\left[\phi_{1}(\zeta, \eta), \phi_{2}(\zeta, \eta), \ldots, \phi_{K_{p}}(\zeta, \eta)\right],
$$

where $K_{p}$ is the number of degrees of freedom on the standard element.

For the model problem (2.1), let $E=\{e\}$ be a finite element mesh of the domain $\Omega$. In the $p$-version, the set $E$ consists of a finite number of elements, and will not change throughout the solution process. The basis functions defined on domain $\Omega$, which are based on the standard basis functions $\phi$ and the finite element mesh $E$, are denoted by

$$
\Phi=\left[\Phi_{1}(x, y), \Phi_{2}(x, y), \ldots, \Phi_{N_{p}}(x, y)\right],
$$

where $N_{p}$ is the number of degrees of freedom for the global problem.

Let $V_{p} \subset H(\Omega)$ be the $p$-version of the finite element space spanned by the polynomial basis functions $\Phi$.

The finite element solution in the $p$-version. Find a function $u \in V_{p} \subset$ $H(\Omega)$ such that

$$
a(u, v)=f(v) \quad \forall v \in V_{p} .
$$

From the $V$-ellipticity assumption, it is easy to see that there exist positive constants $c$ and $C$ such that

$$
c \tilde{a}(u, u) \leq a(u, u) \leq C \tilde{a}(u, u),
$$


where

$$
\tilde{a}(u, v)=\int_{\Omega}(\nabla u \cdot \nabla v+u v) d x .
$$

Define

$$
\tilde{V}_{p}=H^{1}(\Omega) \cap P_{p}(\Omega),
$$

where $P_{p}(\Omega)$ is the space of piecewise polynomials of degree $\leq p$.

$\tilde{V}_{p}$ may be regarded as the finite element space with only Neumann boundary conditions. It is easy to see that $V_{p} \subset \tilde{V}_{p}$. Analysis is easier over $\tilde{V}_{p}$ than over $V_{p}$, since the elemental stiffness matrices over $\tilde{V}_{p}$ all have the same size, provided the $p$-level is uniformly distributed, whereas this may not be the case over the space $V_{p}$ due to possible Dirichlet boundary conditions.

Let $\left\{\Phi_{i}\right\}_{i=1}^{N_{p}}$ be the basis functions of $V_{p}$ and $\left\{\Phi_{i}\right\}_{i=1}^{\tilde{N}_{p}}$ be the basis functions of $\tilde{V}_{p}$. Obviously, $N_{p} \leq \tilde{N}_{p}$. The stiffness matrix based on $a(u, u)$ over $V_{p}$ is defined by

$$
A=\left(a_{i j}\right)=\left(a\left(\Phi_{i}, \Phi_{j}\right)\right),
$$

and the stiffness matrix based on $\tilde{a}(u, u)$ over $\tilde{V}_{p}$ is defined by

$$
\tilde{A}=\left(\tilde{a}_{i j}\right)=\left(\tilde{a}\left(\Phi_{i}, \Phi_{j}\right)\right) \text {. }
$$

It is easy to show that

$$
c \lambda_{\min }(\tilde{A}) \leq \lambda_{\min }(A) \leq \lambda_{\max }(A) \leq C \lambda_{\max }(\tilde{A}) .
$$

Therefore, we have

Lemma 2.1. There exists a constant $C$, independent of the mesh and p-level, such that

$$
\kappa(A) \leq C \kappa(\tilde{A}) .
$$

Remark 1. For the rest of this paper, we consider only the bilinear form $\tilde{a}(u, v)$ over $\tilde{V}_{p}$ and the corresponding stiffness matrix $\tilde{A}$. But for the simplicity of notation, we will use $a(u, v), V_{p}$, and $A$ to stand for $\tilde{a}(u, v), \tilde{V}_{p}$, and $\tilde{A}$, respectively.

On the standard element $K$, we define the stiffness matrix by

$$
A_{K}=\left(a_{i j}\right)_{K}=\left(a\left(\phi_{i}, \phi_{j}\right)\right) .
$$

Let a pair of mapping functions between an element $e \in E$ and the standard element $K$ be denoted by

$$
\left\{\begin{array}{l}
x=Q_{x}^{(e)}(\zeta, \eta) \\
y=Q_{y}^{(e)}(\zeta, \eta)
\end{array}\right.
$$

and the Jacobian matrix by $J_{e}(\zeta, \eta)$.

A basic assumption for mapping functions (2.18) is that their inverses exist. In practice, we can assume that the Jacobian matrix is nonsingular on $K$, which is equivalent to

$$
\operatorname{det}\left(J_{e}(\zeta, \eta)\right) \neq 0 \text { for any }(\zeta, \eta) \in K \text {. }
$$


In general, the determinant $\operatorname{det}\left(J_{e}\right)$ is a continuous function on $K$; therefore, there exist two constants $c_{e} \geq \tilde{c}_{e}>0$ such that

$$
\tilde{c}_{e} \leq\left|\operatorname{det}\left(J_{e}\right)\right| \leq c_{e} \text { for every }(\zeta, \eta) \in K .
$$

Let

$$
C_{e}=\max _{(\zeta, \eta) \in K} \lambda_{\max }\left(\left(J_{e} J_{e}^{T}\right)^{-1}\right)
$$

and

$$
\tilde{C}_{e}=\min _{(\zeta, \eta) \in K} \lambda_{\min }\left(\left(J_{e} J_{e}^{T}\right)^{-1}\right) .
$$

Then, since $K$ is a compact set in $R^{2}$, we have

$$
0<\tilde{C}_{e} \leq C_{e}<\infty \text {. }
$$

Let $A_{e}$ be the elemental stiffness matrix, then, the stiffness matrix $A$ can be assembled from the elemental stiffness matrices by

$$
A=\sum_{e \in E} M_{e} A_{e} M_{e}^{T}
$$

where the matrix $M_{e}$ is the $N_{p} \times K_{p}$ transformation matrix. Let $x \in R^{N_{p}}$, define

$$
x_{e}=M_{e}^{T} x,
$$

then, $x_{e} \in R^{K_{p}}$.

To derive the bounds, we first need to establish a relationship between elemental stiffness matrices and the stiffness matrix on the standard square element.

It is easy to show (see [12] for details) that for each element $e \in E$, we have

$$
\tilde{c}_{e}^{\prime}\left(A_{K} x_{e}, x_{e}\right) \leq\left(A_{e} x_{e}, x_{e}\right) \leq c_{e}^{\prime}\left(A_{K} x_{e}, x_{e}\right),
$$

where $c_{e}^{\prime}=\max \left\{C_{e} c_{e}, c_{e}\right\}$ and $\tilde{c}_{e}^{\prime}=\min \left\{\tilde{C}_{c} \tilde{c}_{e}, \tilde{c}_{e}\right\}$.

By using (2.26) and the Rayleigh quotient, one can show [12] the following relationships of eigenvalues and condition numbers between stiffness matrices on the general domain $\Omega$ and on the standard element $K$.

Theorem 2.1. For a stiffness matrix $A$ on the general domain $\Omega$, its maximum eigenvalue is bounded by

$$
\tilde{c}_{e} \lambda_{\max }\left(A_{K}\right) \leq \lambda_{\max }(A) \leq c_{E} \lambda_{\max }\left(A_{K}\right),
$$

where $\tilde{c}_{E}=\max _{e \in E}\left\{\tilde{c}_{e}^{\prime}\right\}, c_{E}=\sum_{e \in E} c_{e}^{\prime}$; its minimum eigenvalue is bounded by

$$
\tilde{c}_{E}^{\prime} \lambda_{\min }\left(A_{K}\right) \leq \lambda_{\min }(A),
$$

where $\tilde{c}_{E}^{\prime}=\min _{e \in E}\left\{\tilde{c}_{e}^{\prime}\right\}$; and, therefore, its condition number is bounded by

$$
\kappa(A) \leq \hat{c}_{E}^{\prime} \kappa\left(A_{K}\right),
$$

where $\hat{c}_{E}^{\prime}=\frac{c_{E}}{\tilde{c}_{E}^{\prime}}$.

Remark 2. The inequalities analogous to (2.27), (2.28) and (2.29) also hold for the mass matrices $M$ and $M_{K}$, where

$$
M=\left(m_{i j}\right), m_{i j}=\int_{\Omega} \Phi_{i} \Phi_{j} d x
$$

and $M_{K}$ is the corresponding mass matrix defined on the standard element $K$. 
Remark 3. The constants in bounds of (2.27), (2.28) and (2.29) depend only on the topology of the problem domain, the mesh and the mapping functions, but are independent of the differential operator and the basis functions. The eigenvalues of $\lambda_{\max }\left(A_{K}\right)$ and $\lambda_{\min }\left(A_{K}\right)$ depend only on the differential operator and the basis functions.

In Section 3, we derive bounds for the eigenvalues and condition number of $A$. In Section 4, we focus our attention on the stiffness matrix $A_{K}$ on the standard element.

\section{Bounds FOR THE EIGENVALUES AND CONDITION NUMBER IN THE $p$-VERSION}

Let $V_{p}$ be the finite element space spanned by the basis functions $\phi$. We define eigenvalues and eigenvectors of $a(u, u)$ in the finite element space $V_{p}$ as follows [4].

Associated with each space $V_{p}$ are eigenvalues $\lambda_{i}^{(p)}$ and eigenvectors $\Psi_{i}^{(p)} \in V_{p}$, $1 \leq i \leq K_{p}$, satisfying

$$
a\left(\Psi_{i}^{(p)}, v\right)=\lambda_{i}^{(p)}\left(\Psi_{i}^{(p)}, v\right) \quad \text { for all } v \in V_{p}
$$

Without loss of generality, we can assume

$$
0<\lambda_{1}^{(p)} \leq \lambda_{2}^{(p)}<\ldots \leq \lambda_{K_{p}}^{(p)}
$$

For convenience, we will drop all the superscripts $(p)$ from now on. Note that based on the above convention we have $\lambda_{\max }=\lambda_{K_{p}}$ and $\lambda_{\min }=\lambda_{1}$. Then, it is well known that

$$
\lambda_{\max }=\max _{u \in V_{p}-\{0\}} \frac{a(u, u)}{(u, u)}
$$

and

$$
\lambda_{\min }=\min _{u \in V_{p}-\{0\}} \frac{a(u, u)}{(u, u)} .
$$

Let

$$
u=\sum_{j} x_{j} \phi_{j}
$$

then, it is easy to see that

$$
\begin{aligned}
& a(u, u)=(A x, x), \\
& (u, u)=(M x, x) .
\end{aligned}
$$

The relationships among the maximum and minimum eigenvalues of $a(u, u), A$ and $M$ are as follows [12]:

$$
\lambda_{\max }(a) \lambda_{\min }(M) \leq \lambda_{\max }(A) \leq \lambda_{\max }(a) \lambda_{\max }(M)
$$

and

$$
\lambda_{\min }(a) \lambda_{\min }(M) \leq \lambda_{\min }(A) \leq \lambda_{\min }(a) \lambda_{\max }(M) .
$$

Therefore, it follows easily that

$$
\frac{\kappa(a)}{\kappa(M)} \leq \kappa(A) \leq \kappa(a) \kappa(M) .
$$

Now, we want to find bounds for the eigenvalues of the bilinear form $a(u, u)$. In order to derive bounds for the eigenvalues of the bilinear form, we need some 
properties of Legendre polynomials. The following two properties of Legendre polynomials are well known [19]:

$$
\begin{aligned}
& \int_{-1}^{1} P_{m}(x) \cdot P_{n}(x) d x=\left\{\begin{array}{cc}
0 & \text { if } m \neq n \\
\frac{2}{2 n+1} & \text { if } m=n
\end{array},\right. \\
& P_{n}^{\prime}(x)=\sum_{k=0}^{n-2 k-1 \geq 0}(2 n-4 k-1) P_{n-2 k-1}(x) .
\end{aligned}
$$

Based on these two properties, one can show [12] that

$$
\int_{-1}^{1}\left[P_{n}^{\prime}(x)\right]^{2} d z=n(n+1)
$$

We also need the following important inequality.

Schmidt's inequality. For a polynomial $f$ of degree not greater than $N$, the following inequality holds (see [1] and [8]):

$$
\int_{-1}^{1}\left[\frac{\partial f(x)}{\partial x}\right]^{2} d x \leq \frac{(N+1)^{4}}{2} \int_{-1}^{1}[f(x)]^{2} d x .
$$

Theorem 3.1. The maximum eigenvalue $\lambda_{\max }(a)$ of the bilinear form in $V_{p}$ is bounded by

$$
c_{1} p^{3} \leq \lambda_{\max }(a) \leq c_{2} p^{4}
$$

where $c_{1}>0$ and $c_{2}>0$ are constants independent of $p$.

Proof. Without loss of generality, we can assume that the problem domain is the standard element $\Omega=K$ [12]. For the left-hand inequality, by choosing Legendre polynomials $P_{p}\left(x_{1}\right)$ as particular functions for $u$, we have

$$
\lambda_{\max }(a)=\max _{u \neq 0, u \in V_{p}} \frac{a(u, u)}{(u, u)} \geq \frac{a\left(P_{p}, P_{p}\right)}{\left(P_{p}, P_{p}\right)} .
$$

Since

$$
\begin{aligned}
a\left(P_{p}, P_{p}\right) & =\int_{-1}^{1} \int_{-1}^{1}\left(\nabla P_{p} \cdot \nabla P_{p}+P_{p}^{2}\right) d x_{1} d x_{2} \\
& =2 \int_{-1}^{1}\left(\frac{\partial P_{p}}{\partial x_{1}} \frac{\partial P_{p}}{\partial x_{1}}+P_{p}^{2}\right) d x_{1} \\
& \geq 2 \int_{-1}^{1}\left[P_{p}^{\prime}\left(x_{1}\right)\right]^{2} d x_{1},
\end{aligned}
$$

by using the properties of Legendre polynomials, we have

$$
\lambda_{\max }(a) \geq p(p+1)(2 p+1) \geq c_{1} p^{3} .
$$

For the right-hand side inequality, for each fixed $x_{2}, u_{p}\left(x_{1}, x_{2}\right)$ is a polynomial of $x_{1}$; therefore, by using Schmidt's inequality, we have

$$
\int_{-1}^{1}\left[\frac{\partial}{\partial x_{1}} u_{p}\left(x_{1}, x_{2}\right)\right]^{2} d x_{1} \leq c p^{4} \int_{-1}^{1} u_{p}^{2}\left(x_{1}, x_{2}\right) d x_{1} .
$$


By using (3.13), we get

$$
\begin{aligned}
\int_{K} \nabla u_{p} \cdot \nabla u_{p} d x & =\int_{-1}^{1} \int_{-1}^{1}\left[\left(\frac{\partial u_{p}}{\partial x_{1}}\right)^{2}+\left(\frac{\partial u_{p}}{\partial x_{2}}\right)^{2}\right] d x_{1} d x_{2} \\
& \leq c p^{4} \int_{-1}^{1} \int_{-1}^{1} u_{p}^{2}\left(x_{1}, x_{2}\right) d x_{1} d x_{2}
\end{aligned}
$$

Thus, we obtain

$$
a\left(u_{p}, u_{p}\right) \leq c_{2} p^{4} \int_{K} u_{p}^{2} d x_{1} d x_{2}
$$

or

$$
a\left(u_{p}, u_{p}\right) \leq c_{2} p^{4}\left(u_{p}, u_{p}\right)
$$

Therefore,

$$
\lambda_{\max }\left(a_{k}\right)=\max _{x \in V_{p}-\{0\}} \frac{a\left(u_{p}, u_{p}\right)}{\left(u_{p}, u_{p}\right)} \leq c_{2} p^{4} .
$$

For the minimum eigenvalue of the bilinear form, we have

Theorem 3.2. The minimum eigenvalue $\lambda_{\min }(a)$ of the bilinear form in $V_{p}$ is bounded by

$$
c_{3} \leq \lambda_{\min }(a) \leq c_{4},
$$

where $c_{4} \geq c_{3}>0$ are constant, independent of $p$.

Proof. For the left-hand inequality, by using the $V$-ellipticity (2.5), we have

$$
\begin{aligned}
\lambda_{\min }(a) & =\min _{u \in V_{p}-\{0\}} \frac{a(u, u)}{(u, u)} \\
& =\min _{u \in V_{p}-\{0\}} \frac{\|u\|_{H^{1}(\Omega)}^{2}}{(u, u)_{H^{0}(\Omega)}} \\
& \geq \min _{u \in V_{p}-\{0\}} \frac{\|u\|_{H^{0}(\Omega)}^{2}}{(u, u)_{H^{0}(\Omega)}} \\
& =1 .
\end{aligned}
$$

Let $0<c_{3} \leq 1$, then

$$
\lambda_{\min }(a) \geq c_{3} .
$$

For the right-hand inequality, by choosing a special polynomial $u\left(x_{1}, x_{2}\right)=x_{1}$, we have

$$
\lambda_{\min }(a)=\min _{u \in V_{p}-\{0\}} \frac{a(u, u)}{(u, u)} \leq \frac{a(u, u)}{(u, u)}
$$

and

$$
\begin{aligned}
a(u, u) & =\int_{\Omega}\left(\nabla u \cdot \nabla u+u^{2}\right) d x \\
& =\int_{\Omega} d x_{1} d x_{2}+\int_{\Omega} x_{1}^{2} d x_{1} d x_{2} \\
& \leq|\Omega|+\tilde{C}|\Omega|,
\end{aligned}
$$


where

$$
\tilde{C}=\max _{x \in \bar{\Omega}} x^{2}
$$

Similarly,

$$
(u, u)=\int_{\Omega} u^{2} d x_{1} d x_{2}=\int_{\Omega} x_{1}^{2} d x_{1} d x_{2} \leq \tilde{C}|\Omega|
$$

Define

$$
c_{4}=\frac{1+\tilde{C}}{\tilde{C}}
$$

then, the desired result follows.

Remark 4 . It is easy to see that the above proofs can be extended straightforwardly to $R^{d}, d \geq 1$ arbitrary. This means that the inequality (3.12) and (3.14) hold for the model problem (2.1) in $R^{d}, d \geq 1$ arbitrary.

By using (2.27), (3.5) and (3.6), we get

Theorem 3.3. For a stiffness matrix $A$ on domain $\Omega$, its maximum eigenvalue can be bounded by

$$
c_{1} \tilde{c}_{E}^{\prime} p^{3} \lambda_{\min }(M) \leq \lambda_{\max }(A) \leq c_{2} c_{E} p^{4} \lambda_{\max }(M),
$$

and its minimum eigenvalue can be bounded by

$$
c_{3} \tilde{c}_{E}^{\prime} \lambda_{\min }(M) \leq \lambda_{\min }(A) .
$$

Therefore, the condition number can be bounded by

$$
\kappa(A) \leq \hat{C}_{E} p^{4} \kappa(M)
$$

where $\hat{C}_{E}=\frac{c_{2} c_{E}}{c_{3} \tilde{c}_{E}^{\prime}}$, independent of $p$.

Remark 5. We can also derive a lower bound for the condition number of the stiffness matrix [12]:

$$
\hat{C}_{E}^{\prime} \frac{p^{3}}{\kappa(M)} \leq \kappa(A)
$$

where $\hat{C}_{E}^{\prime}=\frac{c_{1} \tilde{c}_{E}^{\prime}}{c_{4} c_{E}}$.

Note that Theorem 3.3 is valid in particular when $\Omega$ is $K$, the standard element. When orthonormal basis functions on $K$ are used, we get $M=I$; thus, from Theorem 3.3, and Theorem 2.1 we have

Corollary 3.1. For a set of basis functions which are orthonormal on $K \subset R^{d}$, $d \geq 1$, the condition number of stiffness matrix $A$ on $\Omega$ can be bounded independent of dimension $d$

$$
\kappa(A) \leq C p^{4},
$$

where the constant is independent of $p$.

The result in Corollary 3.1 is analogous to a well-known result in the $h$-version, where, under uniform or quasi-uniform mesh, the condition numbers are bounded by $O\left(h^{-2}\right)$, independent of the dimensions of the spatial domain of the problem [15]. 
Example. Consider $\Omega=K$ and a set of basis functions consisting of normalized Legendre polynomials on the interval $[-1,1]$. Because this set of basis functions is orthonormal, then, from (3.20) and $(3.21), c p^{3} \leq \kappa(A) \leq C p^{4}$. In [22], a similar result for this set of basis functions was also obtained by a detailed estimation.

Remark 6 . The example above indicates that the bounds in (3.20) and (3.21) for the normalized Legendre polynomial basis functions are quite tight, but it is easy to find other basis functions for which the bounds given by (3.18)-(3.21) are not tight. For example, for a set of hierarchical basis functions that will be discussed in Section 4, in the case of 1-D problems on the standard element $[-1,1]$, it can be shown (see Section 4) that $\kappa(M) \leq C p^{4}$, and we get $c p^{-1} \leq \kappa(A) \leq C p^{8}$ from Theorem 3.3; but it can be shown (see Section 4 ) that $\kappa(A)$ is constant, independent of $p$.

Remark 7 . The above discussion indicates that it is probably not the best choice in 1-D to choose basis functions that are orthonormal, in the sense of the magnitude of condition numbers. However, if we choose orthonormal basis functions for both 2-D and 3-D problems, we have $\kappa(A) \leq C p^{4}$, independent of dimensions. Therefore, Theorem 3.3 can be used as a guide in designing basis functions in higher dimensions. A good balance between orthonormality of basis functions and issues such as numerical stability, hierarchical structure, easy implementations, etc. is essential for designing new, efficient basis functions in higher dimensions.

Remark 8. Note that

$$
\kappa(M) \leq \hat{c}_{E} \kappa\left(M_{K}\right)
$$

(see Remark 2). Therefore, to get an upper bound of the condition number of the stiffness matrix $A$, we need to estimate $\lambda_{\max }\left(M_{K}\right)$ and $\lambda_{\min }\left(M_{K}\right)$ only, where $M_{K}$ is the mass matrix on the standard element. Once a set of basis functions is chosen for the standard element, $M_{K}$ can be computed offline once for all $p=$ $1,2 \ldots$ Therefore, $\lambda_{\max }\left(M_{K}\right), \lambda_{\min }\left(M_{K}\right)$ and $\kappa\left(M_{K}\right)$ can also be computed for $p=1,2, \ldots$, and are independent of the underlying problem. For general basis functions $\phi=\left\{\phi_{i}(\zeta, \eta)\right\}$, by using the Schwartz inequality, we have the following upper bounds for $\lambda_{\max }\left(M_{K}\right)$ :

$$
\lambda_{\max }\left(M_{K}\right) \leq\left[\sum_{i=1}^{K_{p}}\left\|\phi_{i}\right\|_{L^{2}}\right] \max _{1 \leq j \leq K_{p}}\left\|\phi_{j}\right\|_{L^{2}}
$$

and

$$
\lambda_{\max }\left(M_{K}\right) \leq\left[\sum_{i=1}^{K_{p}}\left\|\phi_{i}\right\|_{L^{2}}\right]^{2}
$$

\section{EXPLICIT BOUNDS BASED ON A SET OF HIERARCHICAL BASIS FUNCTIONS}

In the previous sections, the bounds for the minimum eigenvalues, maximum eigenvalues and condition numbers are derived based on general basis functions. Because of this generality, it is understandable that, for certain types of basis functions, the bounds given above may be quite loose. Thus, it is possible to derive better bounds for a specific set of basis functions by exploiting its particular 


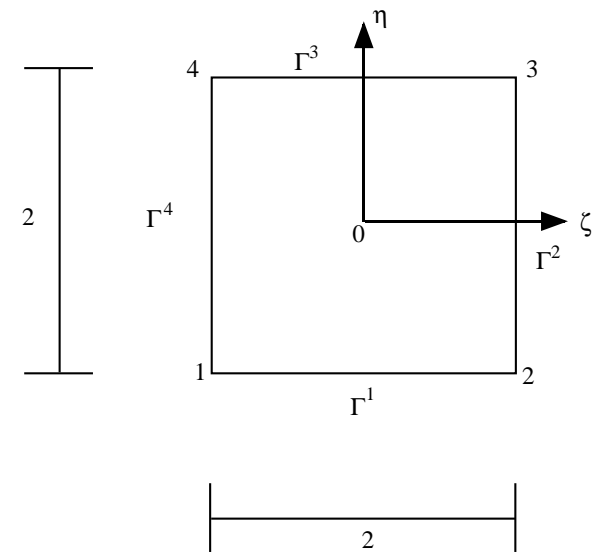

FIGURE 1. The standard quadrilateral element

structure or properties. In this section, we focus our attention on a set of special hierarchical basis functions and derive explicit and quite sharp bounds for the minimum eigenvalues, maximum eigenvalues and condition numbers [13].

Here, we discuss briefly a class of hierarchical basis functions that has been popularly used in the $p$-version. We only present the hierarchical basis functions associated with quadrilateral elements.

Let $K=[-1,1] \times[-1,1]$ be a standard quadrilateral element as shown in Figure 1.

There are four nodal shape functions associated with each vertex:

$$
\begin{aligned}
N^{(1)}(\zeta, \eta) & =\frac{1}{4}(1-\zeta)(1-\eta), \\
N^{(2)}(\zeta, \eta) & =\frac{1}{4}(1+\zeta)(1-\eta), \\
N^{(3)}(\zeta, \eta) & =\frac{1}{4}(1+\zeta)(1+\eta), \\
N^{(4)}(\zeta, \eta) & =\frac{1}{4}(1-\zeta)(1+\eta) .
\end{aligned}
$$

For $p \geq 2$, there are $p-1$ shape functions associated with each side:

$$
\begin{aligned}
S_{i}^{[1]}(\zeta, \eta) & =\frac{1}{2}(1-\eta) \varphi_{i}(\zeta), \\
S_{i}^{[2]}(\zeta, \eta) & =\frac{1}{2}(1+\zeta) \varphi_{i}(\eta), \\
S_{i}^{[3]}(\zeta, \eta) & =\frac{(-1)^{i}}{2}(1+\eta) \varphi_{i}(\zeta), \\
S_{i}^{[4]}(\zeta, \eta) & =\frac{(-1)^{i}}{2}(1-\zeta) \varphi_{i}(\eta), \\
i & =2,3, \ldots, p,
\end{aligned}
$$

where

$$
\varphi_{j}(\zeta)=\sqrt{\frac{2 j-1}{2}} \int_{-1}^{\zeta} P_{j-1}(t) d t
$$


and $P_{j-1}(t)$ is the Legendre polynomial of degree $j-1, j=2,3, \ldots, p$.

Internal shape functions:

$$
\begin{aligned}
I^{(1)}= & \varphi_{2}(\zeta) \varphi_{2}(\eta), \\
I^{(2)}= & \varphi_{3}(\zeta) \varphi_{2}(\eta), \\
I^{(3)}= & \varphi_{2}(\zeta) \varphi_{3}(\eta), \\
I^{(4)}= & \varphi_{4}(\zeta) \varphi_{2}(\eta), \\
I^{(5)}= & \varphi_{3}(\zeta) \varphi_{3}(\eta), \\
I^{(6)}= & \varphi_{2}(\zeta) \varphi_{4}(\eta), \\
& \text { etc. }
\end{aligned}
$$

For the so-called trunk space [20], for $p \geq 4$, there are $(p-2)(p-3) / 2$ internal shape functions. For the so-called product space [20], for $p \geq 2$ there are $(p-1)^{2}$ internal shape functions.

This set of basis functions is hierarchic because the finite element space $V_{p-1}$, which is spanned by the above polynomial basis functions with degree up to $p-1$, is completely embedded into the space $V_{p}$ which is spanned by the above basis functions with degree up to $p$. If we define

$$
\varphi_{0}(t)=\frac{1-t}{2}, \quad \varphi_{1}(t)=\frac{1+t}{2},
$$

then the basis functions defined above can be expressed as

$$
\Phi_{i j}(\zeta, \eta)=\varphi_{i}(\zeta) \varphi_{j}(\eta), \quad i \geq 0, j \geq 0
$$

with sign adjustment for the side basis functions on sides 3 and 4 .

In general, for a given integer $d \geq 1$, a class of hierarchical basis functions on the standard element $K=[-1,1]^{d}$ can be defined as

$$
\begin{aligned}
& \Phi_{i j \ldots l}\left(x_{i}, x_{j}, \ldots x_{l}\right)=\varphi_{i}\left(x_{i}\right) \varphi_{j}\left(x_{j}\right) \ldots \varphi_{l}\left(x_{l}\right), \\
& i, j, \ldots, l=0,1, \ldots, p .
\end{aligned}
$$

After any type of ordering, we can denote the above basis functions as

$$
\phi=\left[\phi_{1}(x), \phi_{2}(x), \ldots, \phi_{K_{p}}(x)\right] .
$$

For simplicity, we drop the subscript $K$ in this section. Define

$$
S=\int_{K}\left(\nabla(\phi(x))^{T}(\nabla \phi(x)) d x .\right.
$$

We call $S$ the derivative matrix. We have

$$
A=S+M,
$$

where $A$ is the stiffness matrix and $M$ is the mass matrix.

Let $R^{K_{p}}$ be a vector space, where $K_{p}$ is the number of degrees of freedom of the stiffness matrix $A$. Let $U_{p}$ be a subspace of $R^{K_{p}}$ consisting of those vectors such that their components corresponding to the basis function $\Phi_{00 \ldots 0}$ are zero. $U_{p}$ can be interpreted as a subspace of $R^{K_{P}}$ by eliminating a vertex variable. Define

$$
\tilde{\lambda}_{\min }(S)=\min _{0 \neq x \in U_{p}} \frac{x^{T} S x}{x^{T} x} .
$$

The following theorem is our main result of this section. 
Theorem 4.1. For the hierarchical basis functions of the product space, there exist positive constants $c$ and $C$, independent of $p$, such that

$$
\begin{gathered}
c \leq \lambda_{\max }(M) \leq C, \\
c p^{-4 d} \leq \lambda_{\min }(M), \\
c \leq \lambda_{\max }(S) \leq C, \\
\lambda_{\min }(S)=0, \\
c p^{-4(d-1)} \leq \tilde{\lambda}_{\min }(S), \\
c \leq \lambda_{\max }(A) \leq C, \\
c p^{-4(d-1)} \leq \lambda_{\min }(A),
\end{gathered}
$$

where $d \geq 1$ is the number of dimensions.

Remark 9. Our discussions are restricted to the standard element $K$, but the results are valid for the general model problem (2.1) on a general domain $\Omega$ due to Lemma 2.1 and Theorem 2.1 .

Before proving the above theorem, we first derive some results using (4.23). Define $\lambda_{1}(S)$ to be the second smallest eigenvalue or the minimum nonzero eigenvalue of $S$. We have lower bound for $\lambda_{1}(S)$ as follows.

Corollary 4.1. There exists a positive constant $c$, independent of $p$, such that

$$
\lambda_{1}(S) \geq c p^{-4(d-1)} .
$$

Proof. Recall that $U_{p}$ is a subspace of $R^{K_{p}}$ with dimensions $K_{p}-1$. By the CourantFisher minimax theorem,

$$
\begin{aligned}
\lambda_{1}(S) & =\max _{\operatorname{dim}(V)=K_{p}-1} \min _{0 \neq x \in V} \frac{x^{T} S x}{x^{T} x} \\
& \geq \min _{o \neq x \in U_{p}} \frac{x^{T} S x}{x^{T} x} \\
& \geq \tilde{\lambda}_{\min }(S) \\
& \geq c p^{-4(d-1)} .
\end{aligned}
$$

If we define

$$
\kappa(S)=\frac{\lambda_{\max }(S)}{\lambda_{1}(S)},
$$

then we have the upper bounds of the condition numbers as follows.

Corollary 4.2. There exists a positive constant $C$, independent of $p$, such that

$$
\begin{aligned}
\kappa(M) & \leq C p^{4 d}, \\
\kappa(S) & \leq C p^{4(d-1)}, \\
\kappa(A) & \leq C p^{4(d-1)},
\end{aligned}
$$

where $d \geq 1$ is the number of dimensions.

The following lemma pertains to the lower bounds of the condition numbers. 
Lemma 4.1. There exists a positive constant $c$, independent of $p$, such that

$$
\begin{gathered}
c p^{4 d} \leq \kappa(M), \\
c p^{4(d-1)} \leq \kappa(S), \\
c p^{4(d-1)} \leq \kappa(A),
\end{gathered}
$$

where $d \geq 1$ is the number of dimensions.

Proof. Let $M_{I}, S_{I}$ and $A_{I}$ denote submatrices of $M, S$ and $A$ corresponding to the internal basis functions, respectively. It has been proved in [16], [17] that there exist positive constants $\bar{c}$ and $\bar{C}$ such that

$$
\begin{gathered}
\bar{c} p^{4 d} \leq \kappa\left(M_{I}\right) \leq \bar{C} p^{4 d}, \\
\bar{c} p^{4(d-1)} \leq \kappa\left(S_{I}\right) \leq \bar{C} p^{4(d-1)} .
\end{gathered}
$$

By Poincare's inequality, it is easy to see that

$$
c \kappa\left(S_{I}\right) \leq \kappa\left(A_{I}\right) \leq C \kappa\left(S_{I}\right) .
$$

Thus from (4.34) we get

$$
\bar{c} p^{4(d-1)} \leq \kappa\left(A_{I}\right) \leq \bar{C} p^{4(d-1)} .
$$

Note

$$
\begin{aligned}
\kappa\left(M_{I}\right) & \leq \kappa(M), \\
\kappa\left(S_{I}\right) & \leq \kappa(S), \\
\kappa\left(A_{I}\right) & \leq \kappa(A) .
\end{aligned}
$$

Thus from (4.33)-(4.35), the proof is completed.

Theorem 4.1 contains only lower bounds for the minimum eigenvalues. We can easily get upper bounds of the minimum eigenvalues as follows.

Corollary 4.3. There exists a positive constant $C$, independent of $p$, such that

$$
\begin{gathered}
\lambda_{\min }(M) \leq C p^{-4 d}, \\
\lambda_{1}(S) \leq C p^{-4(d-1)}, \\
\lambda_{\min }(A) \leq C p^{-4(d-1)} .
\end{gathered}
$$

Proof. We prove only (4.36) and the proofs of (4.37) and (4.38) are analogous. From Theorem 4.1

$$
\lambda_{\max }(M) \leq C
$$

From Lemma 4.1,

$$
\kappa(M) \geq c p^{4 d}
$$

Thus

$$
\lambda_{\min }(M)=\frac{\lambda_{\max }}{\kappa(M)} \leq \frac{C}{c p^{4 d}}=C p^{-4 d}
$$


TAble 1. Maximum eigenvalues of $S, M$ and $A$

\begin{tabular}{|c|c|c|c|c|c|c|c|c|c|}
\hline$p$ & \multicolumn{3}{|c|}{$1-\mathrm{D}$} & \multicolumn{3}{c|}{$2-\mathrm{D}$} & \multicolumn{3}{|c|}{$3-\mathrm{D}$} \\
\hline & $S$ & $M$ & $A$ & $S$ & $M$ & $A$ & $S$ & $M$ & $A$ \\
\hline 1 & 1.0 & 1.0 & 1.3333 & 1.0 & 1.0 & 1.0 & 1.0 & 1.0 & 1.3333 \\
\hline 2 & 1.0 & 1.3506 & 1.8110 & 1.7225 & 1.8242 & 2.93631 & 2.8251 & 2.4638 & 4.5702 \\
\hline 3 & 1.0 & 1.3506 & 1.8110 & 1.7225 & 1.8242 & 2.93631 & 2.8251 & 2.4638 & 4.5702 \\
\hline 4 & 1.0 & 1.3510 & 1.8126 & 1.7246 & 1.824 & 2.9404 & 2.8305 & 2.4660 & 4.5784 \\
\hline 5 & 1.0 & 1.3510 & 1.8126 & 1.7246 & 1.824 & 2.9404 & 2.8305 & 2.4660 & 4.5787 \\
\hline 6 & 1.0 & 1.3510 & 1.8126 & 1.7246 & 1.824 & 2.9404 & 2.8305 & 2.4660 & 4.5787 \\
\hline 7 & 1.0 & 1.3510 & 1.8126 & 1.7246 & 1.824 & 2.9404 & 2.8305 & 2.4660 & 4.5787 \\
\hline 8 & 1.0 & 1.3510 & 1.8126 & 1.7246 & 1.824 & 2.9404 & 2.8305 & 2.4660 & 4.5787 \\
\hline 9 & 1.0 & 1.3510 & 1.8126 & 1.7246 & 1.824 & 2.9404 & 2.8305 & 2.4660 & 4.5787 \\
\hline 10 & 1.0 & 1.3510 & 1.8126 & 1.7246 & 1.824 & 2.9404 & 2.8305 & 2.4660 & 4.5787 \\
\hline 11 & 1.0 & 1.3510 & 1.8126 & 1.7246 & 1.824 & 2.9404 & 2.8305 & 2.4660 & 4.5787 \\
\hline 12 & 1.0 & 1.3510 & 1.8126 & 1.7246 & 1.824 & 2.9404 & 2.8305 & 2.4660 & 4.5787 \\
\hline 13 & 1.0 & 1.3510 & 1.8126 & 1.7246 & 1.824 & 2.9404 & 2.8305 & 2.4660 & 4.5787 \\
\hline 14 & 1.0 & 1.3510 & 1.8126 & 1.7246 & 1.824 & 2.9404 & - & - & - \\
\hline 15 & 1.0 & 1.3510 & 1.8126 & 1.7246 & 1.824 & 2.9404 & - & - & - \\
\hline 16 & 1.0 & 1.3510 & 1.8126 & 1.7246 & 1.824 & 2.9404 & - & - & - \\
\hline 17 & 1.0 & 1.3510 & 1.8126 & 1.7246 & 1.824 & 2.9404 & - & - & - \\
\hline 18 & 1.0 & 1.3510 & 1.8126 & 1.7246 & 1.824 & 2.9404 & - & - & - \\
\hline 19 & 1.0 & 1.3510 & 1.8126 & 1.7246 & 1.824 & 2.9404 & - & - & - \\
\hline 20 & 1.0 & 1.3510 & 1.8126 & 1.7246 & 1.824 & 2.9404 & - & - & - \\
\hline
\end{tabular}

Remark 10. Corollary 4.2 and Lemma 4.1 imply that the condition numbers of $S$ and $A$ are equivalent to $p^{4(d-1)}$ while the condition number of $M$ is equivalent to $p^{4 d}$. Similarly, Theorem 4.1 and Corollary 4.3 imply that the minimum eigenvalues of $S$ and $A$ are equivalent to $p^{-(d-1)}$ while the minimum eigenvalue of $M$ is equivalent to $p^{-4 d}$.

We next present some numerical evidence to verify that the bounds for the minimum and maximum eigenvalues are correct. Table 1 reports the maximum eigenvalues of $S, M$ and $A$ in 1-D, 2-D and 3-D for various $p$ levels, which indicates that the maximum eigenvalues of $S, M$ and $A$ are constants, independent of $p$. Assuming that the minimum eigenvalues decay like $c p^{\alpha}$, we estimate the decay factor $\alpha$, using the following formula:

$$
\alpha=\frac{\log \left(\lambda_{\min }^{(p)} / \lambda_{\min }^{(p-1)}\right)}{\log (p /(p-1))},
$$

and Table 2 reports the decay factor $\alpha$ of $S, M$ and $A$ in 1-D, 2-D and 3-D for various $p$ levels. From Table 2, we can see that the minimum eigenvalues of $S$ and $A$ decay like $c p^{-4(d-1)}$ and the minimum eigenvalues of $M$ decay like $c p^{-4 d}$. These numerical results are very consistent with and support the theoretical results.

The proof of Theorem 4.1 is divided into three parts. In part 1, we prove everything that is related to the mass matrix $M$. In the second part, we focus on the matrix $S$. In the part 3, we prove the results in Theorem 4.1 for the stiffness matrix $A=S+M$. The proofs are given only for $d=2$ and proofs for other dimensions are analogous. 
TABle 2. Decay factors $\alpha$ of minimum eigenvalues of $M, S$ and $A$

\begin{tabular}{|c|c|c|c|c|c|c|c|c|c|}
\hline & \multicolumn{2}{|c|}{$1-\mathrm{D}$} & \multicolumn{3}{c|}{$2-\mathrm{D}$} & \multicolumn{3}{|c|}{$3-\mathrm{D}$} \\
\hline & $S$ & $M$ & $A$ & $S$ & $M$ & $A$ & $S$ & $M$ & $A$ \\
\hline$p$ & $\alpha$ & $\alpha$ & $\alpha$ & $\alpha$ & $1 \alpha$ & $\alpha$ & $\alpha$ & $\alpha$ & $\alpha$ \\
\hline 2 & 0 & -2.755 & 0.7636 & -3.1065 & -5.5111 & -3.4096 & -5.9427 & -8.2667 & -6.0940 \\
\hline 3 & 0 & -1.8290 & 0 & -1.2290 & -3.6580 & 1.0623 & -2.9201 & -5.4871 & -2.0921 \\
\hline 4 & 0 & -3.2198 & -0.0083 & -3.6139 & -6.4378 & -3.6910 & -6.8738 & -9.6568 & -6.8948 \\
\hline 5 & 0 & -2.4316 & 0 & -1.9223 & -4.8632 & -1.8632 & -4.3012 & -7.2949 & -4.3010 \\
\hline 6 & 0 & -3.4884 & 0 & -3.8458 & -6.9769 & -3.8782 & -7.3552 & -10.465 & -7.3611 \\
\hline 7 & 0 & -2.7890 & 0 & -2.3663 & -5.5780 & -2.3395 & -5.6686 & -8.3670 & -5.1313 \\
\hline 8 & 0 & -3.6342 & 0 & -3.9481 & -7.2685 & -3.9647 & -7.5940 & -10.902 & -7.5963 \\
\hline 9 & 0 & -3.0188 & 0 & -2.6630 & -6.0376 & -2.6487 & -5.6686 & -9.0565 & -5.6690 \\
\hline 10 & 0 & -3.7199 & 0 & -3.9961 & -7.4399 & -4.0057 & -7.7232 & -11.159 & -7.7247 \\
\hline 11 & 0 & -3.1170 & 0 & -2.8716 & -6.3540 & -2.8632 & -6.0408 & -9.5311 & -6.0411 \\
\hline 12 & 0 & -3.7749 & 0 & -4.0202 & -7.5499 & -4.0262 & -7.7998 & -11.325 & -7.8001 \\
\hline 13 & 0 & -3.2919 & 0 & -3.0253 & -6.5838 & -3.0199 & -6.3122 & -9.876 & -6.3125 \\
\hline- & - & - & - & - & - & - & - & - & - \\
\hline 19 & 0 & -3.5019 & 0 & -3.3100 & -7.0038 & -3.3081 & - & - & - \\
\hline 20 & 0 & -3.8769 & 0 & -4.0437 & -7.7538 & -4.0452 & - & - & - \\
\hline 21 & 0 & -3.8897 & 0 & -3.3715 & -7.0938 & -3.3700 & - & - & - \\
\hline 22 & 0 & -3.5844 & 0 & -4.0439 & -7.7794 & -4.04511 & - & - & - \\
\hline 23 & 0 & -3.9001 & 0 & -3.4230 & -7.1689 & -3.4219 & - & - & - \\
\hline 24 & 0 & -3.6163 & 0 & -4.0435 & -7.8003 & -4.0444 & - & - & - \\
\hline 25 & 0 & -3.9088 & 0 & -3.4668 & -7.2327 & -3.4659 & - & - & - \\
\hline 26 & 0 & -3.6437 & 0 & -4.0427 & -7.8176 & -4.0434 & - & - & - \\
\hline 27 & 0 & -3.9160 & 0 & -3.5045 & -7.2874 & -3.5038 & - & - & - \\
\hline 28 & 0 & -3.6674 & 0 & -4.0416 & -7.8321 & -4.0423 & - & - & - \\
\hline 29 & 0 & -3.9222 & 0 & -3.5372 & -7.3349 & -3.5367 & - & - & - \\
\hline 30 & 0 & -3.6883 & 0 & -4.0405 & -7.8444 & - & - & - & - \\
\hline$\infty$ & 0 & -4 & 0 & -4 & -8 & -4 & -8 & -12 & -8 \\
\hline
\end{tabular}

\subsection{Bounds for mass matrix $M$.}

Lemma 4.2. There exists a positive integer $N Z$ independent of $p$, such that the number of nonzero entries in each row of $M$ is less than or equal to $N Z$.

Proof. Consider $d=2$. Define

$$
J(i, j)=\int_{-1}^{1} \varphi_{i}(t) \varphi_{j}(t) d t .
$$

Note that

$$
\begin{aligned}
& J(0, j)=\left\{\begin{array}{cc}
2 / 3 & \text { if } j=0 \\
1 / 3 & \text { if } j=1 \\
-1 / \sqrt{6} & \text { if } j=2 \\
1 /(3 \sqrt{10}) & \text { if } j=3 \\
0 & \text { if } j>3,
\end{array}\right. \\
& J(1, j)=\left\{\begin{array}{cc}
2 / 3 & \text { if } j=1 \\
-1 / \sqrt{6} & \text { if } j=2 \\
1 /(3 \sqrt{10}) & \text { if } j=3 \\
0 & \text { if } j>3,
\end{array}\right.
\end{aligned}
$$




$$
J(i, j)=\left\{\begin{array}{cl}
2 /((2 i+1)(2 i-3)) & \text { if } j=i, i>1 \\
0 & \text { if } j=i+1, i>1 \\
-1 /((2 i+1) \sqrt{(2 i+1)(2 i+3)}) & \text { if } j=i+2, i>1 \\
0 & \text { if } j>i+2, i>1 .
\end{array}\right.
$$

Thus,

$$
J(i, j)=0 \quad \text { if }|i-j|>3 .
$$

Given any two basis functions

$$
\Phi_{i j}(\zeta, \eta)=\varphi_{i}(\zeta) \varphi_{j}(\eta)
$$

and

$$
\Phi_{m n}(\zeta, \eta)=\varphi_{m}(\zeta) \varphi_{n}(\eta)
$$

we have

$$
\int \Phi_{i j}(\zeta, \eta) \Phi_{m n}(\zeta, \eta) d \zeta d \eta=J(i, m) \times J(j, n)=0
$$

if $|i-m|>3$ or $|j-n|>3$. In other words, there are at most $7^{d}(d=2$ in this case) nonzeros for each row of $M$.

Lemma 4.3. Let

$$
M=\left(m_{i j}\right) .
$$

There exists a constant $C$, independent of $p$, such that for all $i, j$

$$
\left|m_{i j}\right| \leq C
$$

Proof. Consider $d=2$. From (4.40)-(4.42), we have

$$
|J(i, j)| \leq \frac{2}{3}
$$

Given any two basis functions

$$
\Phi_{i j}(\zeta, \eta)=\varphi_{i}(\zeta) \varphi_{j}(\eta)
$$

and

$$
\Phi_{m n}(\zeta, \eta)=\varphi_{i}(\zeta) \varphi_{j}(\eta)
$$

we get

$$
\left|\int \Phi_{i j}(\zeta, \eta) \Phi_{m n}(\zeta, \eta) d \zeta d \eta\right|=|J(i, m) \times J(j, n)| \leq \frac{4}{9}
$$

Lemma 4.4. There exist constants $c$ and $C$, independent of $p$, such that

$$
c \leq \lambda_{\max }(M) \leq C .
$$

Proof. The left hand side inequality follows by noting that at least one diagonal term of $M$ is constant, independent of $p$. The right hand side inequality follows from Lemmas 4.2-4.3 and the Gershgorin circle theorem [14].

Lemma 4.5. There exists a constant $C$, independent of $p$, such that

$$
\lambda_{\min }(M) \geq C p^{-4 d} .
$$


Proof. Consider $d=2$. Let $\Phi_{i j}(\zeta, \eta)$ be the basis functions defined above. We now define

$$
\tilde{\Phi}_{i j}(\zeta, \eta)=\tilde{P}_{i}(\zeta) \tilde{P}_{j}(\eta),
$$

where $\tilde{P}$ is the normalized Legendre polynomial. Let $B$ be the transformation matrix from $\tilde{\Phi}$ to $\Phi$ :

Since

$$
\int_{K} \tilde{\Phi} \tilde{\Phi}^{T}=I
$$

thus

$$
M=\int_{K} \Phi \Phi^{T}=B\left(\int_{K} \tilde{\Phi} \tilde{\Phi}^{T}\right) B^{T}=B B^{T} .
$$

Noting that

$$
\lambda_{\min }(M)=\frac{1}{\lambda_{\max }\left(M^{-1}\right)},
$$

we next prove that there exists a constant $C$, independent of $p$, such that

$$
\lambda_{\max }\left(M^{-1}\right) \leq C p^{8} .
$$

Since

$$
\varphi_{i}(\zeta)=\frac{1}{\sqrt{2(2 i-1)}}\left(\sqrt{\frac{2}{2 i+1}} \tilde{P}_{i}(\zeta)-\sqrt{\frac{2}{2 i-3}} \tilde{P}_{i-2}(\zeta)\right)
$$

thus

$$
\begin{aligned}
\tilde{P}_{i}(\zeta)= & \sqrt{\frac{2 i+1}{2 i-3}} \tilde{P}_{i-2}(\zeta)+\sqrt{(2 i-1)(2 i+1)} \phi_{i}(\zeta) \\
= & \sqrt{\frac{2 i+1}{2 i-3}}\left(\sqrt{\frac{2 i-3}{2 i-7}} \tilde{P}_{i-4}(\zeta)+\sqrt{(2 i-5)(2 i-3)} \phi_{i-2}\right) \\
& +\sqrt{(2 i-1)(2 i+1)} \phi_{i}(\zeta) \\
= & \sqrt{\frac{2 i+1}{2 i-7}} \tilde{P}_{i-4}(\zeta)+\sqrt{(2 i+1)(2 i-5)} \varphi_{i-2}+\sqrt{(2 i-1)(2 i+1)} \varphi_{i}(\zeta) \\
= & \cdots .
\end{aligned}
$$

In summary, for even $i=2 j$,

$$
\tilde{P}_{i}(\zeta)=\sqrt{2 i+1} \tilde{P}_{0}(\zeta)+\sqrt{2 i+1} \sum_{k=1}^{j} \sqrt{4 k-1} \varphi_{2 k}(\zeta)
$$

and for odd $i=2 j+1$,

$$
\tilde{P}_{i}(\zeta)=\sqrt{\frac{2 i+1}{3}} \tilde{P}_{0}(\zeta)+\sqrt{2 i+1} \sum_{k=1}^{j} \sqrt{4 k+1} \varphi_{2 k+1}(\zeta) .
$$

It follows that there exists a constant $C$, independent of $i$, for $0 \leq i \leq p$, such that

$$
\tilde{P}_{i}(\zeta)=\sum_{k=0}^{i} c_{k}^{i} \varphi_{k}(\zeta)
$$


where $\left|c_{k}^{i}\right| \leq C p$ for all $k$. Thus

$$
\tilde{\Phi}_{i j}(\zeta, \eta)=\sum_{k=0}^{i} c_{k}^{i} \varphi_{k}(\zeta) \sum_{l=0}^{j} c_{l}^{j} \varphi_{l}(\eta)=\sum_{k=0}^{i} \sum_{l=0}^{j} c_{k}^{i} c_{l}^{j} \varphi_{k}(\zeta) \varphi_{l}(\eta) .
$$

Let

$$
e_{k l}=c_{k}^{i} c_{l}^{j}
$$

Since

$$
\left|c_{k}^{i}\right| \leq C p, \quad\left|c_{l}^{j}\right| \leq C p
$$

we have

$$
\left|e_{k l}\right| \leq C p^{2}
$$

Note that $B^{-1}$ is the transformation matrix from the base $\Phi$ to $\tilde{\Phi}$ and (4.46) is also a transformation from the base $\Phi$ to $\tilde{\Phi}$. Therefore, by (4.46), if

$$
B^{-1}=\left(\tilde{b}_{i j}\right),
$$

there exists a constant $C$, independent of $p$, such that

$$
\left|\tilde{b}_{i j}\right| \leq C p^{2}
$$

for all $i, j$. Note that

$$
M^{-1}=B^{-T} B^{-1} \text {. }
$$

Since the order of $M$ and $B$ are $K_{p}=O\left(p^{2}\right)$ in 2-d $\left(K_{p}=O\left(p^{d}\right)\right.$ in general), thus, if

$$
M^{-1}=\left(\tilde{m}_{i j}\right), \tilde{m}_{i j}=\sum_{k=1}^{K_{p}} \tilde{b}_{k i} \tilde{b}_{k j},
$$

there exists a constant $C$, independent of $p$, such that $\left|\tilde{m}_{i j}\right| \leq C p^{6}$. By the Gershgorin circle theorem,

$$
\lambda_{\max }\left(M^{-1}\right) \leq C p^{8} .
$$

\subsection{Bounds for matrix $S$.}

Lemma 4.6. There exist constants $c, C$, independent of $p$, such that

$$
c \leq \lambda_{\max }(S) \leq C .
$$

Proof. The proof is analogous to that of Lemma 4.4.

Lemma 4.7. There exists a constant $c$, independent of $p$, such that

$$
\tilde{\lambda}_{\min }(S) \geq c p^{-4(d-1)} .
$$

Proof. Consider $d=2$. Define

$$
\begin{aligned}
S_{\zeta} & =\left(\int_{K} \frac{\partial \phi_{i}}{\partial \zeta} \frac{\partial \phi_{j}}{\partial \zeta} d \zeta d \eta\right) \\
S_{\eta} & =\left(\int_{K} \frac{\partial \phi_{i}}{\partial \eta} \frac{\partial \phi_{j}}{\partial \eta} d \zeta d \eta\right)
\end{aligned}
$$


Clearly,

$$
S=S_{\zeta}+S_{\eta}
$$

Since

$$
\int_{-1}^{1} \varphi_{k}^{\prime}(t) \varphi_{l}^{\prime}(t) d t=\left\{\begin{array}{cl}
\frac{1}{2} & \text { if } k=l=0 \text { or } 1 \\
-1 / 2 & k=0, l=1 \text { or } k=1, l=0 \\
\delta_{k l} & \text { if } k>1 \text { or } l>1
\end{array}\right.
$$

thus, using order $i \times(p+1)+j, S_{\zeta}$ is of block diagonal form

$$
S_{\zeta}=\left[\begin{array}{cccc}
B_{1} & 0 & \cdots & 0 \\
0 & B_{2} & \cdots & 0 \\
\cdots & \cdots & \cdots & \cdots \\
0 & 0 & \cdots & B_{p}
\end{array}\right],
$$

where

$$
\begin{array}{r}
B_{1}=\frac{1}{2}\left[\begin{array}{cc}
M & -M \\
-M & M
\end{array}\right], \\
B_{i}=M, i>1,
\end{array}
$$

and $M$ is the 1-D mass matrix.

Given any vector $x \in R^{K_{p}}$, decompose $x$ into

$$
x=\left(x^{0}, x^{1}, \ldots, x^{p}\right),
$$

where

$$
x^{k}=\left(x_{k, 0}, x_{k, 1}, \ldots, x_{k, p}\right) .
$$

Thus

$$
x^{T} S_{\zeta} x=\frac{1}{2}\left[\left(x^{0}\right)^{T} M x^{0}-2\left(x^{0}\right)^{T} M x^{1}+\left(x^{1}\right)^{T} M x^{1}\right]+\sum_{k=2}^{p}\left(x^{k}\right)^{T} M x^{k},
$$

and we get

$$
x^{T} S_{\zeta} x \geq \frac{1}{2}\left[\left(x^{0}\right)^{T} M x^{0}-2\left(x^{0}\right)^{T} M x^{1}+\left(x^{1}\right)^{T} M x^{1}\right]+\lambda_{\min }(M) \sum_{k=2}^{p}\left(x^{k}\right)^{T} x^{k} .
$$

Since $M$ is symmetric positive definite, there exists an orthogonal matrix $Q$ such that

$$
M=Q^{T} D Q,
$$

where $D$ is a diagonal matrix with the eigenvalues of $M$ as its diagonal terms. Define

$$
y^{k}=Q x^{k}, k=0,1, \ldots, p .
$$

Then

$$
\begin{aligned}
x^{T} S_{\zeta} x & \geq \frac{1}{2}\left[\left(y^{0}\right)^{T} D y^{0}-2\left(y^{0}\right)^{T} D y^{1}+\left(y^{1}\right)^{T} D y^{1}\right]+\lambda_{\min }(M) \sum_{k=2}^{p}\left(y^{k}\right)^{T} y^{k} \\
& \geq \frac{1}{2} \lambda_{\min }(M) \sum_{l=0}^{p}\left(y_{0, l}^{2}+y_{1, l}^{2}-2 y_{0, l} y_{1, l}\right)+\lambda_{\min }(M) \sum_{k-2}^{p} \sum_{l=0}^{p} y_{k, l}^{2} .
\end{aligned}
$$


Since

$$
\begin{aligned}
\sum_{l=0}^{p} x_{k, l}^{2} & =\left(x^{k}\right)^{T} x^{k}=\left(y^{k}\right)^{T} y^{k}=\sum_{l=0}^{p} y_{k, l}^{2}, k=0,1, \ldots, p, \\
\sum_{l=0}^{p} x_{0, l} x_{1, l} & =\left(x^{0}\right)^{T} x^{1}=\left(y^{0}\right)^{T} y^{1}=\sum_{l=0}^{p} y_{0, l} y_{1, l}
\end{aligned}
$$

we get

$$
\begin{aligned}
x^{T} S_{\zeta} x & \geq \frac{1}{2} \lambda_{\min }(M) \sum_{l=0}^{p}\left(x_{0, l}^{2}+x_{1, l}^{2}-2 x_{0, l} x_{1, l}\right)+\lambda_{\min }(M) \sum_{k=2}^{p} \sum_{l=0}^{p} x_{k, l}^{2} \\
& =I X_{1}+I X_{2},
\end{aligned}
$$

where

$$
\begin{aligned}
& I X_{1}=\frac{1}{2} \lambda_{\min }(M) \sum_{l=0}^{p}\left(x_{0, l}^{2}+x_{1, l}^{2}-2 x_{0, l} x_{1, l}\right), \\
& I X_{2}=\lambda_{\min }(M) \sum_{k=2}^{p} \sum_{l=0}^{p} x_{k, l}^{2} .
\end{aligned}
$$

Using the ordering $i+j \times(p+1)$ and the decomposition (4.50)-(4.51), we can similarly prove that

$$
\begin{aligned}
x^{T} S_{\eta} x & \geq \frac{1}{2} \lambda_{\min }(M) \sum_{k=0}^{p}\left(x_{k, 0}^{2}+x_{k, 1}^{2}-2 x_{k, 0} x_{k, 1}\right)+\lambda_{\min }(M) \sum_{k=0}^{p} \sum_{l=2}^{p} x_{k, l}^{2} \\
& =I Y_{1}+I Y_{2},
\end{aligned}
$$

where

$$
\begin{aligned}
& I Y_{1}=\frac{1}{2} \lambda_{\min }(M) \sum_{k=0}^{p}\left(x_{k, 0}^{2}+x_{k, 1}^{2}-2 x_{k, 0} x_{k, 1}\right) \\
& I Y_{2}=\lambda_{\min }(M) \sum_{k=0}^{p} \sum_{l=2}^{p} x_{k, l}^{2} .
\end{aligned}
$$

Therefore,

$$
x^{T} S x=x^{T} S_{\zeta} x+x^{T} S_{\eta} x \geq I X_{1}+I Y_{1}+I X_{2}+I Y_{2} .
$$

Note that the term $I X_{2}$ includes all the components of $x$ except $x_{k, l}$ for $k=0$ or 1 and $l=0,1, \ldots, p$. The term $I Y_{2}$ includes all components of $x$ except $x_{k, l}$ for $k=0,1, \ldots, p$ and $l=0$ or 1 . Thus, $I X_{2}+I Y_{2}$ includes all components of $x$ except $x_{k, l}$ for $k=0$ or 1 and $l=0$ or 1 .

Next, we will prove that for any $x \in U_{p}$,

$$
I X_{1}+I Y_{1} \geq \frac{1}{4} \lambda_{\min }(M) \sum_{k=0}^{1} \sum_{l=0}^{1} x_{k, l}^{2}
$$


To this end, notice

$$
\begin{aligned}
I X_{1}+I Y_{1} & \geq \frac{1}{2} \lambda_{\min }(M) \sum_{l=0}^{1}\left(x_{0, l}^{2}+x_{1, l}^{2}-2 x_{0, l} x_{1, l}\right) \\
& +\frac{1}{2} \lambda_{\min }(M) \sum_{k=0}^{p}\left(x_{k, 0}^{2}+x_{k, 1}^{2}-2 x_{k, 0} x_{k, 1}\right) .
\end{aligned}
$$

Since $x \in U_{p}, x_{0,0}=0$, so

$$
\begin{aligned}
I X_{1}+I Y_{1} \geq & \frac{1}{2} \lambda_{\min }(M)\left(x_{1,0}^{2}+x_{0,1}^{2}+x_{1,1}^{2}-2 x_{0,1} x_{1,1}\right. \\
& \left.+x_{0,1}^{2}+x_{1,0}^{2}+x_{1,1}^{2}-2 x_{1,0} x_{1,1}\right) \\
= & \frac{1}{2} \lambda_{\min }(M)\left(2 x_{0,1}^{2}+2 x_{1,0}^{2}+2 x_{1,1}^{2}-2 x_{0,1} x_{1,1}-2 x_{1,0} x_{1,1}\right) .
\end{aligned}
$$

Using the inequality $2 a b \leq \frac{a^{2}}{s}+s b^{2}$ with $s=\frac{2}{3}$, we get

$$
\begin{aligned}
2 x_{0,1} x_{1,1} & \leq \frac{3}{2} x_{0,1}^{2}+\frac{2}{3} x_{1,1}^{2}, \\
2 x_{1,0} x_{1,1} & \leq \frac{3}{2} x_{1,0}^{2}+\frac{2}{3} x_{1,1}^{2} .
\end{aligned}
$$

Therefore,

$$
\begin{aligned}
I X_{1}+I Y_{1} & \geq \frac{1}{2} \lambda_{\min }(M)\left(\frac{1}{2} x_{0,1}^{2}+\frac{1}{2} x_{1,0}^{2}+\frac{1}{2} x_{1,1}^{2}\right) \\
& =\frac{1}{4} \lambda_{\min }(M)\left(x_{0,1}^{2}+x_{1,0}^{2}+x_{1,1}^{2}\right) .
\end{aligned}
$$

The inequality (4.53) follows from $x_{0,0}=0$ for any $x \in U_{p}$.

So far, we have proved that there exists a constant $c$, independent of $p$, such that

$$
x^{T} S x \geq c \lambda_{\min }(M) x^{T} x
$$

where $M$ is the 1-D mass matrix. The desired inequality follows from (4.20) which has been proved in Section 4.1.

\subsection{Bounds for the stiffness matrix $A$.}

Lemma 4.8. There exist constants $c, C$, independent of $p$, such that

$$
c \leq \lambda_{\max }(A) \leq C .
$$

Proof. The proof follows from the proofs of Lemmas 4.4 and 4.6.

Given any vector $x \in R^{K_{p}}$, define its associated function $u$ by

$$
u=\sum_{i=1}^{K_{p}} x_{i} \phi_{i}(\zeta, \eta) .
$$

Let $w$ be such a vector in $R^{K_{p}}$ associated with $u=1$. 
Lemma 4.9. The function $u$ associated with $x \in R^{K_{p}}$ is constant if and only if $S x=0$.

Proof. Omitted.

Lemma 4.10. There exists a constant $c$, independent of $p$, such that

$$
\lambda_{\min }(A) \geq c p^{-4(d-1)} .
$$

Proof. Consider $d=2$. Let $Q=\left(q^{0}, q^{1}, \ldots, q^{K_{p}-1}\right)$ be the orthogonal matrix consisting of the eigenvectors of $S$ such that $q^{0}$ is the eigenvector associated with the zero eigenvalue. Given any $x$, define $y=Q^{T} x$ with $y=\left(y_{0}, y_{1}, \ldots, y_{K_{p}-1}\right)^{1}$, $x^{0}=y_{0} q^{0}$ and $x^{1}=\sum_{i=1}^{K_{p}-1} y_{i} q^{i}$. Obviously

$$
x^{T} x=y^{T} y, \quad x=x^{0}+x^{1} .
$$

Note that

$$
x^{T} A x=x^{T} S x+x^{T} M x,
$$

and

$$
x^{T} S x=\left(x^{0}\right)^{T} S x^{0}+2\left(x^{1}\right)^{T} S x^{0}+\left(x^{1}\right)^{T} S x^{1} .
$$

Since $\lambda_{0}(S)=0$, we have

$$
S x^{0}=y_{0} S q^{0}=y_{0} \lambda_{0}(S) q^{0}=0
$$

and

$$
\begin{aligned}
x^{T} S x & =\left(x^{1}\right)^{T} S x^{1} \\
& =\left(\sum_{i=1}^{K_{p}-1} y_{i} q^{i}\right)^{T}\left(\sum_{i=1}^{K_{p}-1} y_{i} \lambda_{i}(S) q^{i}\right) \\
& =\sum_{i=1}^{K_{p}-1} y_{i}^{2} \lambda_{i}(S) \\
& \geq \lambda_{1}(S) \sum_{i=1}^{K_{p}-1} y_{i}^{2} .
\end{aligned}
$$

Using Corollary 4.1, we get

$$
x^{T} S x \geq c p^{-4} \sum_{i=1}^{K_{p}-1} y_{i}^{2} .
$$

Let us now turn our attention to $x^{T} M x$. Notice that

$$
x^{T} M x=\left(x^{0}\right)^{T} M x^{0}+2\left(x^{1}\right)^{T} M x^{0}+\left(x^{1}\right)^{T} M x^{1} .
$$

Let $u^{0}$ and $u^{1}$ be the functions associated with $x^{0}$ and $x^{1}$, respectively. Since $S x^{0}=0$, using Lemma 4.9 , we know $u^{0}$ is a constant, i.e. $u^{0}=\alpha$, where $\alpha$ is scalar constant. Thus $x^{0}=\alpha w$ and

$$
\begin{aligned}
x^{T} M x & =\int_{K}\left(u^{0}\right)^{2}+2 \int_{K} u^{0} u^{1}+\int_{K}\left(u^{1}\right)^{2} \\
& =4 \alpha^{2}+2 \int_{K} \alpha u^{1}+\int_{K}\left(u^{1}\right)^{2}
\end{aligned}
$$


where 4 comes from the area of the standard element $K$ in $2-D$. Since for any constant $s>0$,

$$
2 \int_{K} \alpha u^{1} \geq-\left(s \int_{K} \alpha^{2}+\frac{1}{s} \int_{K}\left(u^{1}\right)^{2}\right)=-\left(4 s \alpha^{2}+\frac{1}{s} \int_{K}\left(u^{1}\right)^{2}\right),
$$

we get

$$
x^{T} M x \geq 4(1-s) \alpha^{2}+\left(1-\frac{1}{s}\right) \int_{K}\left(u^{1}\right)^{2} .
$$

Note that, from (4.20),

$$
\int_{K}\left(u^{1}\right)^{2}=\left(x^{1}\right)^{T} M x^{1} \geq \lambda_{\min }(M)\left(x^{1}\right)^{T} x^{1} \geq c p^{-8}\left(x^{1}\right)^{T} x^{1} .
$$

Recall that $x^{1}=\sum_{i=1}^{K_{p}-1} y_{i} q^{i}$. Thus

$$
\int_{K}\left(u^{1}\right)^{2}=\left(x^{1}\right)^{T} M x^{1} \geq c p^{-8} \sum_{i=1}^{K_{p}-1} y_{i}^{2}
$$

and

$$
x^{T} M x \geq 4(1-s) \alpha^{2}+\left(1-\frac{1}{s}\right) c p^{-8} \sum_{i=1}^{K_{p}-1} y_{i}^{2} .
$$

Combining (4.55) and (4.56), we get

$$
x^{T} A x \geq 4(1-s) \alpha^{2}+c\left[p^{-4}+\left(1-\frac{1}{s}\right) p^{-8}\right] \sum_{i=1}^{K_{p}-1} y_{i}^{2} .
$$

Setting $s=\frac{1}{1+p^{-4} / 2}$, we have

$$
x^{T} A x \geq 4\left(\frac{p^{-4} / 2}{1+p^{-4} / 2}\right) \alpha^{2}+c\left(p^{-4}-\frac{p^{-12}}{2}\right) \sum_{i=1}^{K_{p}-1} y_{i}^{2} .
$$

Thus, there exists a positive constant $c$, independent of $p$, such that

$$
x^{T} A x \geq c p^{-4}\left(4 \alpha^{2}+\sum_{i=1}^{K_{p}-1} y_{i}^{2}\right) .
$$

Since $x^{0}=\alpha w$,

$$
y_{0}^{2}=\left(x^{0}\right)^{T} x^{0}=\alpha^{2} w^{T} w=4 \alpha^{2} .
$$

Therefore, there exists a positive constant $c$, independent of $p$, such that

$$
x^{T} A x \geq c p^{-4}\left(y_{0}^{2}+\sum_{i=1}^{K_{p}-1} y_{i}^{2}\right)=c p^{-4} y^{T} y .
$$


TABle 3. Maximum eigenvalues of $S, M$ and $A$ based on trunk space

\begin{tabular}{|c|c|c|c|c|c|c|c|c|c|}
\hline$p$ & \multicolumn{3}{|c|}{$1-\mathrm{D}$} & \multicolumn{3}{c|}{$2-\mathrm{D}$} & \multicolumn{3}{|c|}{$3-\mathrm{D}$} \\
\hline & $S$ & $M$ & $A$ & $S$ & $M$ & $A$ & $S$ & $M$ & $A$ \\
\hline 1 & 1.0 & 1.0 & 1.3333 & 1.0 & 1.0 & 1.333 & 1.0 & 1.0 & 1.3333 \\
\hline 2 & 1.0 & 1.3506 & 1.8110 & 1.459 & 1.693 & 2.261 & 1.850 & 2.033 & 2.6667 \\
\hline 3 & 1.0 & 1.3506 & 1.8110 & 1.459 & 1.693 & 2.261 & 1.850 & 2.033 & 2.6667 \\
\hline 4 & 1.0 & 1.3510 & 1.8126 & 1.7222 & 1.824 & 2.937 & 2.411 & 2.417 & 4.1896 \\
\hline 5 & 1.0 & 1.3510 & 1.8126 & 1.7222 & 1.824 & 2.9937 & 2.411 & 2.417 & 4.1896 \\
\hline 6 & 1.0 & 1.3510 & 1.8126 & 1.7246 & 1.8252 & 2.9404 & 2.465 & 2.4660 & 4.5764 \\
\hline 7 & 1.0 & 1.3510 & 1.8126 & 1.7246 & 1.8252 & 2.9404 & 2.465 & 2.4660 & 4.5764 \\
\hline 8 & 1.0 & 1.3510 & 1.8126 & 1.7246 & 1.8252 & 2.9404 & 2.8305 & 2.4660 & 4.5787 \\
\hline 9 & 1.0 & 1.3510 & 1.8126 & 1.7246 & 1.8252 & 2.9404 & 2.8305 & 2.4660 & 4.5787 \\
\hline 10 & 1.0 & 1.3510 & 1.8126 & 1.7246 & 1.8252 & 2.9404 & 2.8305 & 2.4660 & 4.5787 \\
\hline 11 & 1.0 & 1.3510 & 1.8126 & 1.7246 & 1.8252 & 2.9404 & 2.8305 & 2.4660 & 4.5787 \\
\hline 12 & 1.0 & 1.3510 & 1.8126 & 1.7246 & 1.8252 & 2.9404 & 2.8305 & 2.4660 & 4.5787 \\
\hline 13 & 1.0 & 1.3510 & 1.8126 & 1.7246 & 1.8252 & 2.9404 & 2.8305 & 2.4660 & 4.5787 \\
\hline 14 & 1.0 & 1.3510 & 1.8126 & 1.7246 & 1.8252 & 2.9404 & 2.8305 & 2.4660 & 4.5787 \\
\hline 15 & 1.0 & 1.3510 & 1.8126 & 1.7246 & 1.8252 & 2.9404 & 2.8305 & 2.4660 & 4.5787 \\
\hline 16 & 1.0 & 1.3510 & 1.8126 & 1.7246 & 1.8252 & 2.9404 & 2.8305 & 2.4660 & 4.5787 \\
\hline 17 & 1.0 & 1.3510 & 1.8126 & 1.7246 & 1.8252 & 2.9404 & 2.8305 & 2.4660 & 4.5787 \\
\hline 18 & 1.0 & 1.3510 & 1.8126 & 1.7246 & 1.8252 & 2.9404 & 2.8305 & 2.4660 & 4.5787 \\
\hline 19 & 1.0 & 1.3510 & 1.8126 & 1.7246 & 1.8252 & 2.9404 & 2.8305 & 2.4660 & 4.5787 \\
\hline 20 & 1.0 & 1.3510 & 1.8126 & 1.7246 & 1.8252 & 2.9404 & 2.8305 & 2.4660 & 4.5787 \\
\hline
\end{tabular}

The proof is completed by noting that

$$
x^{T} x=y^{T} y .
$$

The proof of Theorem 4.1 is now complete.

So far, our analysis has been restricted to the set of hierarchical basis functions in the so-called product space. Now, let us consider the trunk space. Since, for the same $p$ level, the basis functions for the trunk space are a subset of the basis functions for the product space, Theorem 4.1 is applicable to the trunk space as well.

The only question is whether the bounds in Theorem 4.1 are still sharp based on the basis functions in the trunk space? In Tables 3 and 4 we provide numerical results for the trunk space, which indicate that the bounds given in Theorem 4.1 are still quite sharp for the trunk space.

From Tables 3 and 4, we can see that the maximum eigenvalues are all constants and for large $p$, they are basically the same as those based on the product space. The minimum eigenvalues are larger in magnitude than the corresponding ones based on the product space, but they decay at the same rates as do those based on the product space. The same can be said about the condition numbers based on the trunk space.

The bounds given in Theorem 4.1 and Corollaries 4.1, 4.2 are similiar to the results in [16], [17]. There are, however, important differences. In [16], [17], basis functions are restricted to internal modes (so-called "Bubble" modes), whereas ours consider all modes. This is essential for practical finite element analysis. Also our results apply to the matrices $S, M$ and $A=S+M$; the results in [16], [17] apply only to $S, M$ separately. 
TABle 4. Decay factors $\alpha$ of the minimum eigenvalues of $S, M$ and $A$ based on trunk space

\begin{tabular}{|c|c|c|c|c|c|c|}
\hline & \multicolumn{3}{|c|}{$2-\mathrm{D}$} & \multicolumn{3}{c|}{$3-\mathrm{D}$} \\
\hline & $S$ & $M$ & $A$ & $S$ & $M$ & $A$ \\
\hline$p$ & $\alpha$ & $\alpha$ & $\alpha$ & $\alpha$ & $\alpha$ & $\alpha$ \\
\hline 2 & -1.2822 & -2.7556 & -1.3956 & -1.5849 & -2.7556 & -1.6619 \\
\hline 3 & 0.0000 & -2.2229 & -0.0041 & -0.3910 & -2.5582 & -0.4645 \\
\hline 4 & -4.3956 & -3.7072 & -4.8470 & -4.4790 & -3.2364 & -4.4478 \\
\hline 5 & -0.7407 & -3.3196 & -0.4117 & -1.2925 & -4.0727 & -1.3573 \\
\hline 6 & -2.7568 & -6.5748 & -3.0311 & -7.1625 & -6.1517 & -7.2718 \\
\hline 7 & -2.4309 & -4.3091 & -2.1738 & -1.9838 & -4.8139 & -1.8764 \\
\hline 8 & -4.1599 & -7.1754 & -4.2895 & -6.3193 & -10.255 & -6.4494 \\
\hline 9 & -2.0999 & -4.9205 & -1.9805 & -3.7383 & -5.8688 & -3.6476 \\
\hline 10 & -4.0468 & -7.4596 & -4.1246 & -7.5008 & -10.749 & -7.5679 \\
\hline 11 & -2.5378 & -5.3758 & -2.4682 & -4.4198 & -6.7169 & -4.3723 \\
\hline 12 & -4.1506 & -7.6223 & -4.2013 & -8.0126 & -11.165 & -8.0477 \\
\hline 13 & -2.7525 & -5.7206 & -2.7075 & -4.7168 & -7.3604 & -4.6951 \\
\hline- & - & - & - & - & - & - \\
\hline 19 & -3.1304 & -6.3733 & -3.1154 & - & - & - \\
\hline 20 & -4.2112 & -7.8759 & -4.2242 & - & - & - \\
\hline 21 & -3.2131 & -6.5162 & -3.2020 & - & - & - \\
\hline 22 & -4.2068 & -7.9012 & -4.2168 & - & - & - \\
\hline 23 & -3.2821 & -6.6363 & -3.2736 & - & - & - \\
\hline 24 & -4.2009 & -7.9203 & -4.2087 & - & - & - \\
\hline 25 & -3.3403 & -6.7386 & -3.3336 & - & - & - \\
\hline 26 & -4.1942 & -7.9350 & -4.2004 & - & - & - \\
\hline 27 & -3.3900 & -6.8268 & -3.3847 & - & - & - \\
\hline 28 & -4.1873 & -7.9466 & -4.1924 & - & - & - \\
\hline 29 & -3.4329 & -6.9305 & -3.4286 & - & - & - \\
\hline 30 & -4.1804 & -7.9559 & -4.1845 & - & - & - \\
\hline$\infty$ & -4 & -8 & -4 & -8 & -12 & -8 \\
\hline & & & & & & \\
\hline
\end{tabular}

\section{Conclusion}

In this paper, bounds are derived for the minimum eigenvalues, maximum eigenvalues and condition numbers of stiffness matrices based on the $p$-version of the finite element method and general basis functions. We present a quite general, yet simple approach to this problem. For a set of hierarchical basis functions that has been popularly used in the $p$-version, explicit bounds are derived for the eigenvalues of the mass matrix $M$, the derivative matrix $S$ and the stiffness matrix $A$. Our results show that the condition number of the stiffness matrices grow like $p^{4(d-1)}$, where $d$ is the number of dimensions. Numerical simulation results are also provided, which verify that our theoretical bounds are correct. Numerical results for condition numbers have been reported by Babuska et al in [6] and [7]. They observed empirically (for $p \leq 8$ ) that the condition numbers grow like $p^{3}$ in 2 -D based on their numerical examples. Our results show theoretically and numerically that in the asymptotic range the condition numbers in 2-D grow like $p^{4}$. Our results in Corollary 4.2 disprove a conjecture in [22] in which the authors assert that "regardless of the choice of basis, the condition numbers grow like $p^{4 d}$ or faster." 


\section{ACKNOWLEDGMENT}

We would like to thank the referee for his thorough and helpful review, which has helped us make this paper much clearer and stronger.

\section{REFERENCES}

1. I. Babuska, B.A. Szabo and I.N. Katz, The p-version of the finite element method, SIAM J. Numer. Anal. Vol. 18, No. 3, June 1981. MR 82j:65081

2. I. Babuska and M. Suri, The optimal convergence rate of the p-version of the finite element methods, SIAM J. Numer. Anal. Vol. 24, No. 4, August 1987. MR 88k:65102

3. I. Babuska and M.Suri, The $p$ - and $h-p$ versions of the finite element method, An overview, Comput. Methods Appl. Mech. Engrg. 80, 5-26, 1990. MR 91j:73064

4. I. Babuska, B. Guo and J.E. Osborn, Regularity and numerical solution of eigenvalue problems with piecewise analytic data, SIAM J. Numer. Anal. vol. 26, pp. 1534-1560, December, 1989. MR 91g:35194

5. I. Babuska, B. Guo and E.P. Stephan, The h-p version of the boundary element method with geometric mesh on polygonal domains, Comput. Methods Appl. Mech. Engrg. 80 (1989), pp. 319-325. MR 91h:65186

6. I. Babuska, A. Craig, J. Mandel and J. Pitkaranta, Efficient preconditionings for the p-version of the finite element method of two dimensions, SIAM J. Numer. Anal., 28 (1991), pp. 624661. MR 92a:65282

7. I. Babuska, M. Griebel and J. Pitkaranta, The problem of selecting the shape functions for a p-type finite element, Internat. J. Numer. Methods Engrg. (1989), pp. 1891-1908. MR 91a:73067

8. R. Bellman, A note on an inequality of E. Schmidt, Bull. Amer. Math. Soc., 50 (1944), pp. 734-736. MR 6:61g

9. R.E. Bank and T. Dupont, An optimal order process for solving finite element equations, Math. Comp. Vol. 36, No. 153, January 1981. MR 82b:65113

10. D. Braess and W. Hackbusch, A new convergence proof of the multigrid method including the V-cycle, SIAM J. Numer. Anal. Vol. 20, No. 5, October 1983. MR 85h:65233

11. J. Bramble and J. E. Pasciak, New convergence estimates for multigrid algorithms, Math. Comp. Vol. 49, No. 180, October 1987. MR 89b:65234

12. N. Hu, Multi-p processes: Iterative algorithms and preconditionings for the p-version of finite element analysis, Doctoral dissertation, Department of Systems Science and Mathematics, Washington University inSt. Louis, August, 1994.

13. N. Hu, X. Guo and I.N. Katz, Lower and upper bounds for eigenvalues and condition numbers in the p-version of the finite element method, SIAM annual meeting, 1995, Charlotte, North Carolina.

14. G. Golub and C. Van Loan, Matrix computations, Second Edition, The Johns Hopkins University Press, Baltimore, 1989. MR 90d:65055

15. C. Johnson, Numerical solution of partial differential equations by the finite element method, Cambridge University Press, Cambridge, New York, 1987. MR 89b:65003a

16. J. F. Maitre and O. Pourquier, Conditionnements et preconditionnements diagonaux des systems pour la p-version des methodes d'elements finis pour des problems elliptiques d'order 2, C. R. Acad. Sci. Paris Sér. I Math. 318 (1994), pp. 583-586. MR 94m:65177

17. J. F Maitre and O. Pourquier, Condition number and diagonal preconditioning: Comparison of the p-version and the spectral element methods, Numer. Math., 74 (1996), pp. 69-84. MR 98b:65047

18. J. T. Marti, Introduction to Sobolev spaces and finite element solution of elliptic boundary value problems, Academic Press Inc., Orlanda, Florida, 1986. MR 89c: 46050

19. G. Sansone, Orthogonal functions, Interscience Publishers, Inc., New York, 1959. MR 21:2140

20. B.A. Szabo and I. Babuska, Finite element analysis, J. Wiley \& Sons, New York, 1991. MR 93f:73001 
21. Stress Check, http://www.esrd.com, Engineering Software Research and Development, Inc., St. Louis, MO 63117, 1994.

22. E.T. Olsen and J. Douglas, Jr., Bounds on spectral condition numbers of matrices arising in the p-version of the finite element method, Numer. Math., 69, 333-352 (1995). MR 95j:65142

Department of Systems Science and Mathematics, Washington University in St. Louis, St. LOUis, MO 63130

Current address: Endocardial Solutions, 1350 Energy Lane, St. Paul, MN 55108

E-mail address: ning@endo.com

Department of Mechanical Engineering, Washington University in St. Louis, St. LOUIS, MO 63130

E-mail address: guo@esrd.com

Department of Systems Science and Mathematics, Washington University in St. Louis, ST. LOUIS, MO 63130

E-mail address: katz@zach.wustl.edu 